\title{
A Behavioral Lifestyle Intervention to Improve Frailty in Overweight or Obese Older Adults with Type 2 Diabetes: A Feasibility Study
}

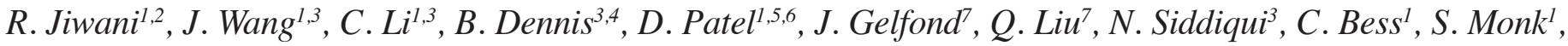 \\ M. Serra ${ }^{2,8,9}$, S. Espinoza $a^{2,8,9}$

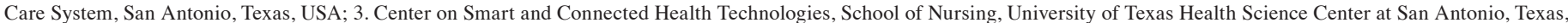

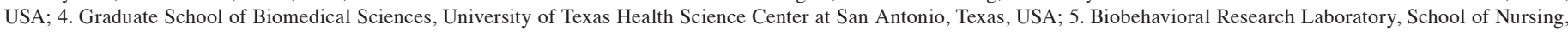

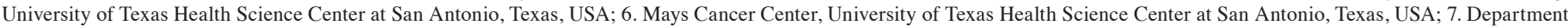

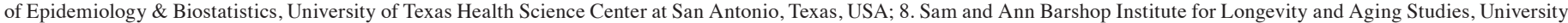

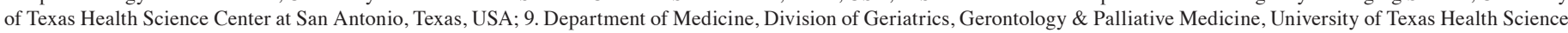
Center at San Antonio, Texas, USA
\end{abstract}

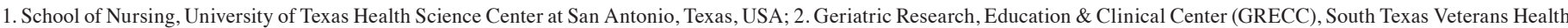

Corresponding Author: Rozmin Jiwani, PhD, RN, 7703 Floyd Curl Drive, San Antonio, Texas, 78229, Phone: 210-450-8498, Fax: 210-567-5822, Jiwani@uthscsa.edu

\begin{abstract}
BACKGROUND: Older adults with Type 2 diabetes (T2D) are more likely to be frail, which increases the risk for disability and mortality.

Objectives: To determine the feasibility of a behavioral lifestyle intervention, enhanced with mobile health technology for selfmonitoring of diet and activity, to improve frailty in overweight/obese older adults ( $\geq 65$ years) diagnosed with T2D.

DESIGN, SETTING, AND PARTICIPANTS: Single arm, 6-month study of a behavioral lifestyle intervention in 20 overweight/obese (BMI $>25$ ) older adults ( $\geq 65$ years) with self-reported T2D diagnosis who owned a smartphone. A Fitbit tracker was provided to all participants for self-monitoring of diet and physical activity. Our primary outcome of feasibility was measured by session attendance, adherence to Fitbit usage to self-monitor diet and physical activity, and study retention. Secondary outcomes included the preliminary efficacy of the intervention on frailty, physical function, quality of life, and T2Drelated outcomes.

RESULTS: Eighteen participants completed the study. The mean age was $71.5(\mathrm{SD} \pm 5.3)$ years, $56 \%$ were female, and half were Hispanic. At baseline, 13 (72\%) were pre-frail, 4 (22\%) were frail, and $1(6 \%)$ were non-frail. At follow-up, frailty scores improved significantly from $1.61 \pm 1.15$ to $0.94 \pm 0.94(\mathrm{p}=0.01)$ and bodyweight improved from $205.66 \pm 45.52 \mathrm{lbs}$. to $198.33 \pm 43.6 \mathrm{lbs}$. $(\mathrm{p}=<0.001)$.

CONCLUSION: This study provides evidence for the feasibility of a behavioral lifestyle intervention in overweight/obese older adults with T2D and preliminary results support its potential efficacy in improving frailty score.
\end{abstract}

Keywords: Type 2 diabetes, lifestyle intervention, self-monitoring, frailty, personal fitness technology.

\section{Introduction}

$\mathrm{F}$ Trailty is characterized as a geriatric syndrome of vulnerability and progressive physical decline, which significantly increases risks for falls, disability, and death (1). Frailty prevalence is approximately $10 \%$ population over 60 years old and reaches over $25 \%$ in those ages 80 years and older (2). Type 2 Diabetes (T2D) and overweight/ obesity are highly prevalent in older adults and are significant Received January 11, 2021

Accepted for publication March 26, 2021 predictors of both onset and worsening of frailty in older adults (3-5). Frailty prevalence is much higher in older adults diagnosed with T2D (32\% to $48 \%$ ) than in the general older population (5\% to $10 \%$ ), and frail individuals with T2D have higher mortality rates (6). Indeed, frailty is emerging as another category of T2D complications, in addition to the traditional micro-and macrovascular sequelae (7). To date, there are no widely accepted evidence-based interventions to improve frailty (8). Such interventions would be especially useful in older adults with T2D as frailty is highly associated with diabetes.

Lifestyle modification is the first-line treatment for T2D and there is evidence to suggest that weight loss and exercise improve components of frailty, such as gait speed, muscle strength, and physical function (8). The Look AHEAD (Action for Health in Diabetes) randomized clinical trial study demonstrated the efficacy of behavioral intensive lifestyle intervention (ILI) of caloric restriction and physical activity, targeting a $7 \%$ weight loss over the control group to reduce adiposity, improve glycemic control (9), reduce risk factors for cardiovascular disease (10), microvascular complications (11), and physical function $(12,13)$ over the follow-up years (range 8-11 years). In addition, ILI participants had less need for diabetes medications (14) and lowered health care costs (15). The ILI intervention was also associated with lower frailty index (FI) scores throughout follow-up (16). The FI operationalizes frailty as deficit accumulation and calculates the proportion of health deficits present in a person. This differs from the Fried frailty phenotype criteria, which scores frailty based on the presence of five criteria, including unintentional weight loss, exhaustion, low physical activity, poor muscle strength, and slow gait speed. The Look AHEAD trial did not assess frailty by Fried phenotype criteria at study baseline or during the intervention but did have this measure at later follow-up examinations. Therefore, the effect of the ILI intervention on frailty characteristics as measured by the Fried criteria is not known.

Self-monitoring is a cornerstone of behavioral lifestyle interventions. The rise in popularity and accessibility of 
personal fitness technology, such as wearable mobile health technology, apps, and mobile devices have become an informative and useful option to positively reinforce health, exercise, and nutritional behavior (17). Technology use has been steadily increasing in older adults, as smartphone ownership in this population has increased from $18 \%$ in 2013 to $43 \%$ in 2017, and the number of older adults who access the internet has risen to $67 \%$ (18). The integration of personal fitness technologies into behavioral interventions has been shown to increase physical activity, decrease sedentary behaviors, and improve objective measures (i.e., glycemic control) in older adults (19-23).

This study aimed to determine the feasibility of Look AHEAD behavioral lifestyle intervention, enhanced with mobile health technology for self-monitoring of diet and physical activity, to improve frailty as assessed by Fried criteria. Our primary outcome of feasibility was measured by session attendance, adherence to Fitbit usage to self-monitor diet and physical activity, and study retention. Secondary outcomes included the preliminary efficacy of the intervention on frailty, physical function, quality of life, and T2D-related outcomes. The primary hypothesis was the study is feasible and behavioral lifestyle intervention will improve frailty in community-dwelling overweight/obese older adults ( $\geq 65$ years) diagnosed with T2D.

\section{Methods}

\section{Study Design}

We conducted a single-arm, 6-month study of a behavioral lifestyle intervention program enhanced with mobile health technology. A Fitbit wristband activity tracker was provided to all participants for self-monitoring of diet and physical activity and the companion app was downloaded on their smartphone by the research team during the baseline screening visit. The study was approved by the Institutional Review Board at the University of Texas Health Science Center at San Antonio (UTHSCSA).

\section{Participant recruitment}

We recruited participants using a combination of methods supported by the San Antonio Claude D. Pepper Older Americans Independence Center, including a call center, community advisory board, and research volunteer registry. We also utilized our University's Find-a-Study recruitment website, advertisement in our primary care clinics, local senior centers, local newspapers, local health fairs/community events, outreach to community leaders, and word of mouth. A study flow diagram using the Consolidated Standards of Reporting Trials (CONSORT) model is shown in Figure 1.
Figure 1. CONSORT flow-diagram for a single arm nonrandomized study

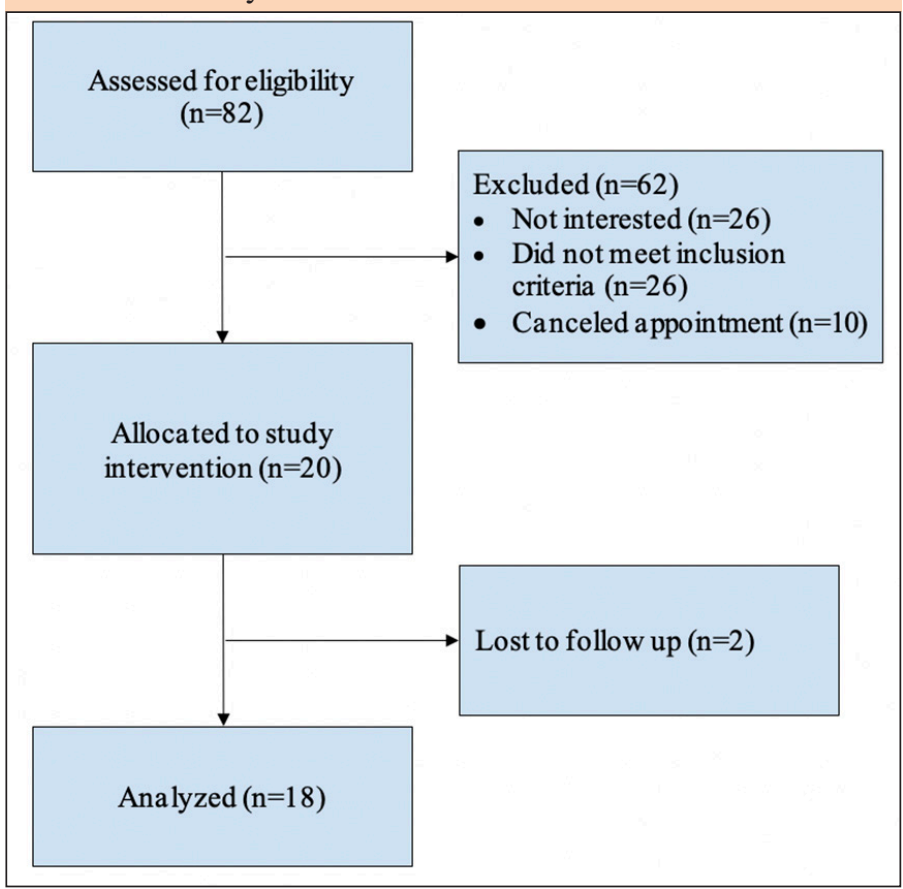

\section{Sample and setting}

We recruited 20, community-dwelling, overweight/obese (BMI $\geq 25 \mathrm{~kg} / \mathrm{m}^{2}$ ), older adults ( $\geq 65$ years) diagnosed with T2D (self-report of provider-diagnosis). We planned that all participants would receive a total of 10 face-to-face group sessions for the behavioral lifestyle intervention over 6 months. However, due to local and institutional COVID-19 restrictions, 3 of our 4 groups (groups 2, 3, and 4) attended 2-3 virtual (WebEx) group educational sessions to complete the study. They were then seen in-person for their end-ofstudy assessments. See Table 1 for detailed inclusion and exclusion criteria. We conducted all the study-related visits at the Biobehavioral Research Laboratory at UTHSCSA School of Nursing.

\section{Sample size}

For this feasibility study, a sample size of $\mathrm{n}=20$ was used, based on Moore et al. (24) which provide recommendations for planning pilot studies in clinical and translational research. We assumed a $40 \%$ attrition rate and enrolled 20 participants.

\section{Study procedures}

Potential participants were informed about the study over the phone and, if interested, proceeded with phone screening for eligibility based on inclusion and exclusion criteria (Table 1). Eligible participants were scheduled for an in-person baseline screening visit. T2D was ascertained by participant self-report of provider-diagnosed T2D in response to the question, "Has a medical doctor or other provider told you that you have Type 2 
Table 1. Inclusion and exclusion criteria

\begin{tabular}{|l|l|}
\hline Inclusion Criteria & Exclusion Criteria \\
\hline $\begin{array}{l}\text { Age } 65 \text { years and older } \\
\text { All ethnic groups }\end{array}$ & Resident of a long-term care facility \\
Self-report of provider diagnosed T2D & History of substance abuse in the past year \\
$\begin{array}{l}\text { Overweight or obese (BMI } \geq 25 \mathrm{~kg} / \mathrm{m} 2) \\
\text { Able to read and write in English }\end{array}$ & Unsafe to walk using Exercise Assessment and Screening for You (EASY) criteria \\
Owns a smartphone & History of severe psychiatric disorders or cognitive impairment \\
\hline
\end{tabular}

diabetes?" We also performed medication review and baseline lab work (fasting blood glucose and Hemoglobin A1c [HbA1c]) to verify the reliability of the self-reported T2D diagnoses. After informed consent, we collected baseline data. These same assessments were repeated at 6-month at the end of the study visit.

\section{Data collection}

Health history and physical assessment: A standardized history and physical assessment were performed and participants' self-reported medical, surgical, and medication history were recorded. We measured participants' vital signs (heart rate, blood pressure, temperature) and anthropometric measurements (waist circumference [inches], height [inches], weight [pounds]. Body mass index was calculated as weight $(\mathrm{kg}) /$ height $\left(\mathrm{m}^{2}\right)$.

Frailty assessment: We classified frailty status using the Fried phenotype criteria (1): 1) Self-reported unintentional weight loss of $\geq 10$ pounds in the past year; 2) Self-reported exhaustion; 3) Low energy expenditure using the Minnesota Leisure Time Physical Activity Questionnaire (MLTQ) to assess physical activity (duration and frequency) (25); 4) Weakness measured via grip strength using a handheld dynamometer in the dominant hand; 5) Timed gait at usual speed over a 10-foot walk as previously described (26). Any intentional weight loss that may have occurred due to the lifestyle intervention would not meet the criteria for frailtyrelated weight loss, as frailty-related weight loss is defined as unintentional. A frailty score was calculated as the number $(0$ -5 ) of frailty characteristics present. Those with $\geq 3$ of these 5 characteristics are categorized as frail; those with 1 or 2 are categorized as pre-frail, and those with none are categorized as non-frail (1).

Physical function: We administered the Short Physical Performance Battery (SPPB), a reliable and valid tool to assess lower extremity physical functions. The SPPB consists of 1) standing balance (ability to stand with the feet together in side-by-side, semi-and full-tandem positions for 10 seconds each); 2) a 4-meter walk (gait speed test) to assess the time it takes to complete the walk at a usual pace; 3 ) time to complete five repeated chair stand without using hands. Each of the 3 performance measures was assigned a score ranging from 0 to 4 and summed to create a score ranging from 0 (worst) to 12 (best). The SPPB is sensitive to change over time $(27,28)$.
Self-reported measures of health and quality of life: Participants completed the Patient-Reported Outcome Measurements Information System (PROMIS-57 and PROMIS Global Health) questionnaires to assess self-reported health and quality of life (29). The PROMIS-57 assesses seven health domains (anxiety, depression, fatigue, pain interference, physical function, satisfaction with participation in social roles, and sleep disturbance) using eight items for each domain. PROMIS Global Health measures are generic, rather than disease-specific, and are intended to globally reflect individuals' assessment of health. The questionnaires are ranked on a 5-point Likert scale and include an additional pain intensity scale (0-10 numeric rating). The feasibility of using PROMIS tools has been supported among ethnic minority, predominantly African American, overweight/obese adults with T2D who had greater symptom burden and poorer physical functioning than the general US population (22).

Clinical laboratory measures: Fasting blood glucose and HbA1c were measured at baseline and at 6-months to monitor safety and the impact of adherence to the interventions. Blood was collected from participants via venipuncture in the antecubital space of the preferred arm. Samples were allowed to sit at room temperature for 30 minutes after which they were centrifuged for 15 mins at 1000xg. Analyses were performed by Quest Diagnostics (Dallas, TX).

\section{Study Intervention}

Behavioral Lifestyle Intervention: We used the publicly available behavioral lifestyle intervention available from the Look AHEAD and Diabetes Prevention Program Group Lifestyle Balance websites in this study. The PI ( $R N, P h D)$ delivered all the sessions. All participants received 10 behavioral lifestyle intervention sessions over 6 months as shown in Table 2. The frequency and design of these sessions (weekly in month 1, biweekly in months 2 and 3, and monthly in months 4 to 6), allowed participants to master new skills gradually, then eventually adopt the behaviors as part of their daily lives (Table 2). Each session lasted 60 to 90 minutes. Missed sessions were replaced with either an individual makeup session or phone consultation depending on the participant's availability. All 10 group sessions focused on adherence to the behavioral strategies, such as self-monitoring, goal setting, feedback, mindful eating, talkback negative thoughts, social support, problem-solving, relapse prevention, and handling 
Table 2. Topics and schedules for group sessions

\begin{tabular}{|c|c|c|}
\hline Baseline Visit & \multicolumn{2}{|c|}{$\begin{array}{l}\text { Data collection, create Fitbit account, connect Fitbit account into a connected health platform, training on the use of the Fitbit activity } \\
\text { tracker, trained on how to record diet and physical activity }\end{array}$} \\
\hline \multirow[t]{3}{*}{ Month 1 (weekly) } & $\begin{array}{l}\text { Wk.1 (Session1): } \\
\text { Welcome to the Program }\end{array}$ & $\begin{array}{l}\text { Participants are given tailored weight loss goals of } 5 \% \\
\text { (1-2 pounds/week), calorie/fat gram recommendations, } \\
\text { and advised to build physical activity to } 175 \text { minutes/ } \\
\text { week by } 6 \text { months }\end{array}$ \\
\hline & $\begin{array}{l}\text { Wk.2 (Session 2): } \\
\text { Tipping the calorie balance \& Be a fat and calorie detective }\end{array}$ & $\begin{array}{l}\text { Cover how to read food labels, and advised to gradually } \\
\text { reduce } 500-1000 \text { calories/week based on the tailored } \\
\text { calorie/fat gram goals to lose } 1-2 \text { pounds/week by } 6 \\
\text { months. }\end{array}$ \\
\hline & $\begin{array}{l}\text { Wk.3 (Session 3): } \\
\text { Move those muscles }\end{array}$ & $\begin{array}{l}\text { Train on how to gradually build up to } 175 \text { minutes/week } \\
\text { of physical activity by } 6 \text { months. }\end{array}$ \\
\hline Month 2 (biweekly) & $\begin{array}{l}\text { Wk.6 (Session 4): } \\
\text { Take charge of what is around you \& Problem-solving \& Healthy eating }\end{array}$ & $\begin{array}{l}\text { Train on how to describe the problem in detail, look for } \\
\text { things that "cue" you (or make you want to overeat or } \\
\text { not be active), and make an action plan to take charge. }\end{array}$ \\
\hline Month 3 (biweekly) & $\begin{array}{l}\text { Wk.12 (Session 7): } \\
\text { Handling holidays and special events }\end{array}$ & $\begin{array}{l}\text { Train on effective ways to handle the holidays, such } \\
\text { as to plan pleasure other than food, and other plans to } \\
\text { handle the holidays effectively. }\end{array}$ \\
\hline Month 4 (monthly) & $\begin{array}{l}\text { Wk.16 (Session 8): } \\
\text { Emotion and you \& Make social cues work for you }\end{array}$ & $\begin{array}{l}\text { Practice responding to emotional and social cues more } \\
\text { healthily. }\end{array}$ \\
\hline Month 5 (monthly) & $\begin{array}{l}\text { Wk.20 (Session 9): } \\
\text { Preventing relapse }\end{array}$ & $\begin{array}{l}\text { Train on effective ways to prevent a relapse by } \\
\text { identifying the lapses early and dealing with them } \\
\text { before they turn into a relapse. }\end{array}$ \\
\hline Month 6 (monthly) & $\begin{array}{l}\text { Wk.24 (Session 10): } \\
\text { Becoming a weight loss expert, Congratulations on completing the program \& } \\
\text { End of study data collection }\end{array}$ & $\begin{array}{l}\text { Encourage the maintenance of changes made in diet } \\
\text { and activity behaviors in order to continue to reach or } \\
\text { maintain previously set goals. }\end{array}$ \\
\hline
\end{tabular}

holidays, among others (Table 2). Participants were given a tailored weight loss goal of 5-7\% based on calorie and fat intake based on their current weight, and physical activity goals to gradually increase to 175 minutes (about 3 hours) per week by the end of the study following Look AHEAD guidelines (9). In every group session, we reinforced the message to adhere to self-monitoring of diet, physical activity, and follow the behavioral education provided during the session. We recorded participants' weight in every in-person group session, but we did not require them to check their weight at home.

\section{Mobile health technology}

A Fitbit wristband activity tracker was provided to all participants at the baseline screening visit, for self-monitoring of diet and activity, and the companion Fitbit app was downloaded on each participants' smartphone. Participants received training on how to record their food (portion size, calories, and fat) and physical activity (duration and type of activity) using the Fitbit wristband activity tracker application on their smartphones. Participants were reminded during group sessions to self-monitor their diet and activity. We entered participants' tailored bodyweight loss and activity goals in their device and encouraged them to record their food and activity daily. We also gave a written/pictorial step-by-step "cheat sheet" of instructions on how to record their food and activity and we reinforced these instructions throughout the study. Participants were asked to wear the device at all times. The research team sent two text messages per month, either for positive feedback or for a gentle reminder to those who were struggling or not recording their diet or physical activity data based on the data gathered from the connected health platform. Adherence to Fitbit usage for physical activity was inferred from step count data. If participants generated step count data for a given day, we determined that the participant had worn the device.

\section{Statistical Analyses}

Feasibility was measured via session attendance, adherence to Fitbit usage to self-monitor diet and physical activity (step count data), and retention at 6 months. We examined the preliminary efficacy of the intervention on frailty phenotype (Fried criteria), including physical function using the Short Physical Performance Battery (SPPB), T2D-related outcomes (body weight, waist circumference, fasting blood glucose, and HbA1c), including self-reported health and quality of life using the Patient-Reported Outcome Measurements Information System (PROMIS) questionnaires. We used a paired t-test to perform the efficacy analysis on the Fried frailty score $(0-5)$, 
Table 3. Participant characteristics $(\mathrm{N}=18)$

\begin{tabular}{|c|c|c|c|}
\hline Characteristic & \multicolumn{3}{|l|}{ Mean (SD) or N (\%) } \\
\hline Age (years) & \multicolumn{3}{|l|}{$71.5(5.3)$} \\
\hline Sex (female) & \multicolumn{3}{|l|}{$10(56 \%)$} \\
\hline Ethnicity & Not Hispanic or Latino & $9(50 \%)$ & \\
\hline T2D duration (years, SD) & \multicolumn{3}{|l|}{$14.4(9.4)$} \\
\hline T2D Treatment $(\%)$ & Combination of oral agents and insulin & $3(17 \%)$ & \\
\hline \multicolumn{2}{|c|}{ Frailty Characteristics at baseline (Yes, \%) } & \multicolumn{2}{|c|}{ Frailty Characteristics at 6-months (Yes, \%) } \\
\hline Slowness & $4(22 \%)$ & Slowness & $2(11 \%)$ \\
\hline Weakness & $9(50 \%)$ & Weakness & $5(27 \%)$ \\
\hline Frailty status at baseline & & Frailty status at 6-months & \\
\hline Pre-Frail & $13(72 \%)$ & Pre-Frail & $9(50 \%)$ \\
\hline Frail & $4(22 \%)$ & Frail & $2(11 \%)$ \\
\hline Non-Frail & $1(6 \%)$ & Non-Frail & $7(39 \%)$ \\
\hline
\end{tabular}

which was selected based upon Type I and II error performance with similar ordinal variables (30). Other exploratory endpoints of frailty, SBBP, and PROMIS component scores were assessed with paired t-tests. The five frailty components (e.g., grip strength, gait speed, physical activity, exhaustion, unintentional weight loss) were analyzed using McNemar's test. Factors (age, gender, and ethnicity) may have moderated intervention effects on outcomes (frailty, body weight, and SPPB) using interaction terms (factor $\mathrm{x}$ time) within linear mixed-effects models. All testing was two-sided with a significance threshold of $<0.05$ for the $\mathrm{p}$-values. All analyses were conducted in $\mathrm{R}$ (v3.5+, Vienna, Austria) within an accountable data analysis process.

\section{Results}

\section{Participant characteristics}

Participant characteristics are detailed in Table 3. A total of 20 participants were enrolled with a mean age of $71.5( \pm 5.3)$ years. Mean BMI was in the obese range (mean $=33.7 \mathrm{~kg} / \mathrm{m} 2 \pm$ 6.3 ), mean frailty score was $1.61 \pm 1.15$ (range: $0-4$ ), and mean SBBP score was $9.22 \pm 2.13$ (range: $7-12$ ) at study baseline. The majority of participants were pre-frail (72\%) at the start of the study.

\section{Feasibility outcome}

Eighteen (90\%) participants completed the study. Two participants dropped out after the first educational session and were no longer interested in participating. Data from the 18 participants who completed the study have been included in all analyses. Of the 18 participants who completed the study, $11(61.1 \%)$ attended all 10 sessions, 6 missed 1 session, and 1 missed 3 sessions. The mean number of sessions attended was 9.5. The median [interquartile range] adherence rate for Fitbit activity tracker device was 95.6\% [79.5\%, 100\%]. The maximum and minimum adherence rates were $100 \%$ and $36.4 \%$, respectively. The median [interquartile range] of diet logging rate (days with at least 1 diet item logged/total days in study) was $81.5 \%[40.7 \%, 84.1 \%]$. For the physical activity logging rate, these numbers were $8.4 \%$ [2.7\%, $48.7 \%]$.

\section{Effect of the study intervention}

Table 4 shows the effect of the study intervention on frailty, physical function, quality of life, and T2D-related outcomes (tailored weight loss, waist circumference, fasting blood glucose, and $\mathrm{HbA1c}$ ). Frailty total score improved significantly by 0.67 points $(95 \%$ CI $[1.15,0.18], \mathrm{p}=0.01)$. Frailty subscores for the 10 -foot timed gait improved significantly at 0.30 points $(95 \%$ CI $[0.61,-0.01], \mathrm{p}=0.05)$, while physical activity and grip strength did not change significantly. Although the SBBP total score did not significantly change, there was a trend toward improvement by 0.83 (95\% CI [0.07, -1.74] $\mathrm{p}=0.069)$. SBBP sub-scores for the balance tests improved significantly 0.50 (95\% CI $[-0.15,-0.85], \mathrm{p}=0.008)$, while gait speed and repeated chair stand scores did not change significantly.

About T2D-related outcomes, on average, participants lost 7.3 lbs. (95\% CI [10.73, 3.92], p<0.001) and reduced their BMI by $1.15 \mathrm{~kg} / \mathrm{m}^{2}$ (95\% CI [1.69, 0.61], p<0.001). Age and ethnicity did not moderate treatment effects $(p>0.05)$. In exploratory analyses, we found that males were more 
Table 4. Effect of the study intervention on frailty, physical function, quality of life, and T2D-related outcomes

\begin{tabular}{|c|c|c|c|c|c|}
\hline Variables & Baseline Mean (SD) & End of study Mean (SD) & Mean Diff & $95 \% \mathrm{CI}$ & p-value \\
\hline Frailty total score & $1.61(1.15)$ & $0.94(0.94)$ & 0.67 & {$[1.15,0.18]$} & 0.01 \\
\hline SPPB total score & $9.22(2.13)$ & $10.06(1.7)$ & -0.83 & {$[0.07,-1.74]$} & 0.069 \\
\hline \multicolumn{6}{|c|}{ Frailty characteristic continuous measures (Timed gait, Physical activity using MLTQ, Grip strength) } \\
\hline Timed gait over10-Feet, sec & $3.68(0.89)$ & $3.38(0.71)$ & 0.30 & {$[0.61,-0.01]$} & 0.053 \\
\hline Physical activity, kcal/week & $1720.83(1354.18)$ & $1673.76(1655.83)$ & 47.07 & {$[746.95,-652.81]$} & 0.889 \\
\hline Grip strength, kg & $24.28(11.88)$ & $26.26(11.34)$ & -1.97 & {$[0.65,-4.6]$} & 0.132 \\
\hline \multicolumn{6}{|c|}{ SPPB sub-score (Balance Tests [side-by-side; semi-tandem, tandem], Gait Speed, Chair Stands) } \\
\hline Balance Tests & $3.39(0.78)$ & $3.89(0.47)$ & -0.50 & {$[-0.15,-0.85]$} & 0.008 \\
\hline Gait Speed & $3.61(0.61)$ & $3.72(0.57)$ & -0.11 & {$[0.18,-0.4]$} & 0.430 \\
\hline Chair Stands & $2.28(1.27)$ & $2.44(1.04)$ & -0.17 & {$[0.43,-0.76]$} & 0.564 \\
\hline \multicolumn{6}{|c|}{ T2D-related outcomes (body weight, waist circumference, fasting blood glucose, HbA1c) } \\
\hline Bodyweight, lbs. & $205.66(45.52)$ & $198.33(43.60)$ & 7.32 & {$[10.73,3.92]$} & $<0.001$ \\
\hline $\mathrm{BMI}, \mathrm{kg} / \mathrm{m}^{2}$ & $33.66(6.28)$ & $32.51(6.40)$ & 1.15 & {$[1.69,0.61]$} & $<0.001$ \\
\hline Waist Circumference, inches & $43.16(4.68)$ & $42.73(4.81)$ & 0.42 & {$[1.36,-0.51]$} & 0.354 \\
\hline Fasting blood glucose, $\mathrm{mg} / \mathrm{dL}$ & $138.81(38.96)$ & $135.63(38.79)$ & 3.18 & {$[18.55,-12.18]$} & 0.665 \\
\hline $\mathrm{HbA} 1 \mathrm{c}, \%$ & $6.95(0.84)$ & $6.75(0.95)$ & 0.2 & {$[0.45,-0.05]$} & 0.108 \\
\hline \multicolumn{6}{|c|}{ PROMIS 57 \& PROMIS Global Health Scores } \\
\hline Anxiety & $47.59(9.95)$ & $49.1(6.09)$ & -1.51 & {$[-6.56,3.55]$} & 0.538 \\
\hline Depression & $45.69(9)$ & $46.76(6.99)$ & -1.07 & {$[-4.36,2.23]$} & 0.504 \\
\hline Fatigue & $51.38(7.43)$ & $50.38(6.51)$ & 1 & {$[-3.48,5.48]$} & 0.643 \\
\hline Pain Interference & $56.08(7.14)$ & $56.63(4.3)$ & -0.55 & {$[-4.18,3.08]$} & 0.753 \\
\hline Physical Function & $43.29(7.54)$ & $43.96(5.92)$ & -0.66 & {$[-3.39,2.06]$} & 0.615 \\
\hline Sleep Disturbance & $59.67(2.47)$ & $59.09(2.21)$ & 0.58 & {$[-1.11,2.26]$} & 0.479 \\
\hline Participate in Social Roles \& Activities & $37.49(6.89)$ & $35.79(5.75)$ & 1.69 & {$[-2.41,5.8]$} & 0.396 \\
\hline Global Physical Health & $42.63(6.37)$ & $44.25(6.77)$ & -1.62 & {$[-4.83,1.59]$} & 0.303 \\
\hline Global Mental Health & $48.87(9.03)$ & $51.69(6.46)$ & -2.82 & {$[-6.38,0.73]$} & 0.112 \\
\hline Pain Intensity & $4.06(1.98)$ & $3.89(1.84)$ & 0.17 & {$[-0.83,1.16]$} & 0.729 \\
\hline
\end{tabular}

responsive to the intervention, with a decrease in weight $(-7.9$ lbs., $\mathrm{p}<0.05)$ and SBBP scores $(2.8, \mathrm{p}<0.001)$ relative to females $(\mathrm{p}<0.05)$. The intervention led to a $3.0 \%$ reduction in $\mathrm{HbA} 1 \mathrm{c}$, a $2.3 \%$ reduction in fasting blood glucose, a $1.0 \%$ reduction in waist circumference, but these changes were not statistically significant. PROMIS scores for self-reported health and quality of life were not affected by the study intervention ( $p$-values $>0.05$ ), however, we noticed some improvements in PROMIS Global Physical and Mental health scores (Table 4). Individual participant's weight for every session is shown in Figure 2, which depicts weight/weight loss normalized by baseline weight. Most of the participants (except for two participants from group one) participated in virtual monthly educational sessions (sessions 8 to 10) due to the local COVID19 quarantine. Seven out of 18 (38\%) participants regained an average of $4.5 \mathrm{lbs}$. of weight during the virtual sessions, even though their weight had appropriately declined before this point (Figure 2).

\section{Discussion}

To our knowledge, this is the first study to determine the feasibility of a behavioral lifestyle intervention, enhanced with mobile health technology for self-monitoring of diet and activity, for the goal of improving frailty in overweight/obese older adults with T2D. We demonstrated a high retention rate with $90 \%$ of participants completing the study, even though we encountered an unexpected obstacle due to the COVID19 pandemic at the latter part of the study. Additionally, our participants were highly adherent to the use of the mobile health technology device (Fitbit) for self-monitoring of diet and activity. Although median adherence was high, we did observe a wide range with the lowest adherence rate of $36.4 \%$, which indicates difficulty by some participants with self-monitoring and/or use of the technology. The procedures for logging diet and physical activity were similar in that they were both entered through the Fitbit app. Step counts were automatically logged when participants wore the Fitbit. We had a high adherence rate for wearing the Fitbit (95.6\%). Although both logging of diet and physical activity was emphasized during group sessions, it is possible that participants could have assumed that since the Fitbit automatically calculates daily step count, it would also automatically detect all other types of physical activity. This could potentially have resulted in a lower logging rate for physical activity. As logging foods is tedious and requires more sophisticated user operations (selecting food items, portions, etc.), the high diet logging rate is encouraging and reaffirms that mobile technology is acceptable in this population. On the 
Figure 2. Individual participants' weight/weight loss normalized by baseline weight. The y-axis is weight percentage of baseline and $\mathrm{x}$-axis is week number at the bottom and session number on the top for behavioral lifestyle intervention

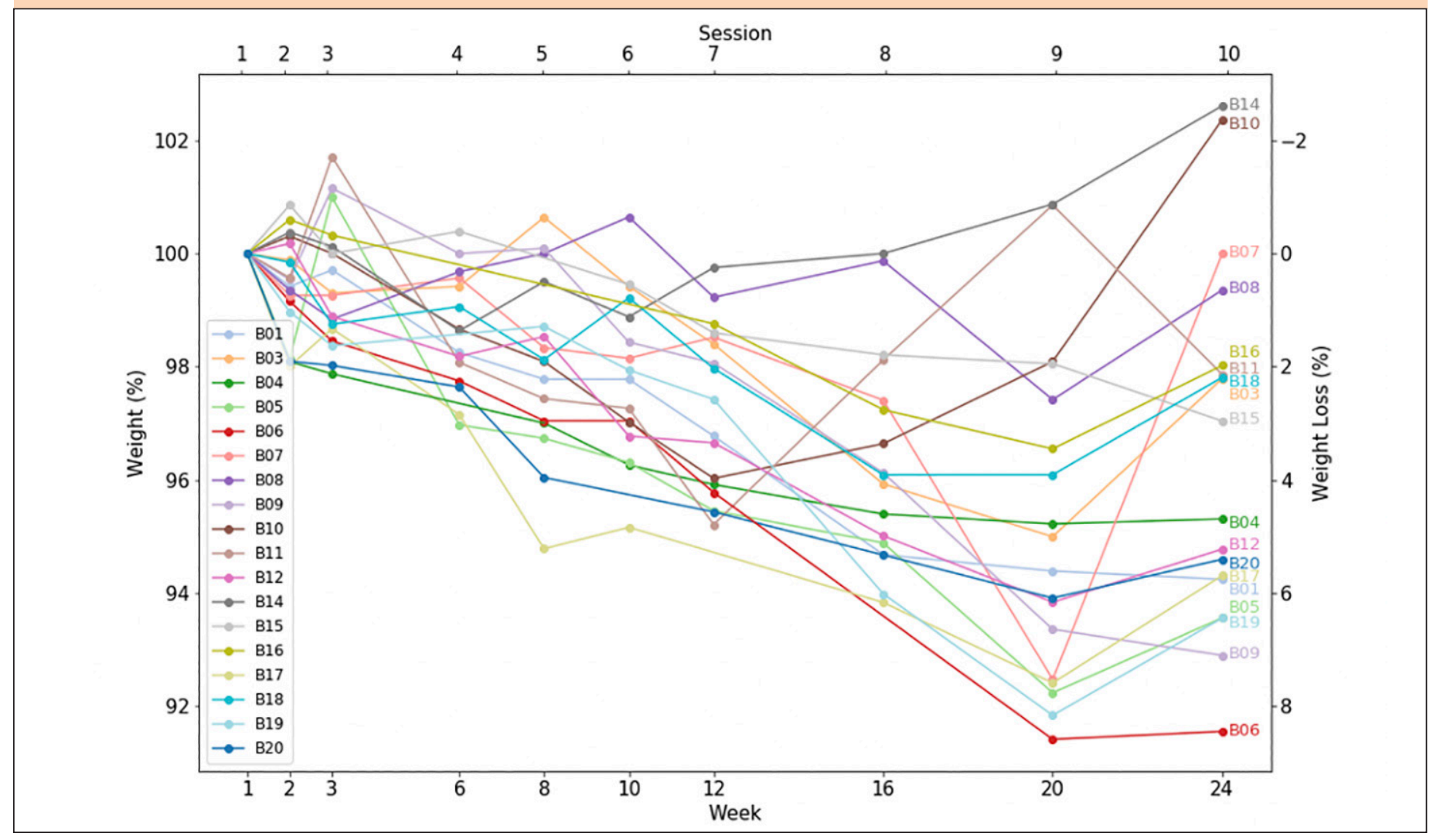

other hand, the low logging rate of physical activity suggests that the intervention may need further improvements to increase physical activity, or that there is a barrier to logging any activities that were performed. Similar findings related to a decrease in self-monitoring adherence in older adults have been reported and authors have suggested that more tailored technology instructions and further engineering may optimize user interface features (31). Future studies might consider providing direct exercise training and/or additional counseling, such as motivational interviewing, on the use of the activity tracker device. In a review of the literature of self-monitoring (diet, exercise, self-weighing) in therapeutic weight loss studies, Burke et al. reported that there was a correlation between adherence to self-monitoring and weight loss. However, there was a gradual decline in self-monitoring adherence throughout the study, which worsened when the treatment sessions decreased in frequency (32). This may, in part, explain our participants' gradual weight increase starting with the monthly group sessions (sessions 8 to 10). However, the weight increase occurred simultaneously with the start of the local COVID-19 pandemic, which also likely impacted progress on weight loss.

The COVID-19 pandemic began during the implementation of this study, and local regulations required shelter-in-place protocols to be implemented throughout the region. Stay-athome orders directly impacted the design of this study and led to our decision to use a virtual format to continue group sessions. This shift affected data collection for some outcomes (such as body weight measurement for every group session) and reduced in-person contact during the latter part of the study, however, we were able to continue using the group format virtually and participants recorded their self-reported weight (recording weight at home was not required for the study) for that session. The COVID-19 pandemic (started from monthly sessions 8-10) also likely had an impact on the behaviors of self-monitoring that we sought to modify with behavioral lifestyle intervention, even though bodyweight had appropriately declined prior to this point (Figure 2). This may also explain non-significant scores for self-reported health and quality of life PROMIS (e.g., anxiety, depression, participation in social activities) questionnaires score. We explored the impact of the COVID-19 experience and acceptability of the behavioral lifestyle intervention in our recently published focus group study wherein participants noted additional challenges to engaging in healthy behaviors as compared to before COVID19 (33).

At baseline, most of our participants were characterized as pre-frail (72\%) and frail (22\%), supporting the high prevalence of frailty in older adults with T2D, as noted in other studies (34). Our pilot findings suggest that the Look AHEAD lifestyle intervention improves frailty characteristics (Table 3), and total frailty score (Table 4) in older adults diagnosed with T2D. In addition, our study participants started with a lower SPPB total score of $9.2 \pm 2.1$. A lower SPPB (score $\leq 9)$ is independently associated with falls (35) and frailty (36). We noticed a significant improvement in the SPPB balance score $(\mathrm{p}=0.008)$. Although there was a positive trend in SPPB total 
score it was not statistically significant $(\mathrm{p}=0.069)$. Similar improvements in SPPB total score have been reported by a 12-month multimodal (diet and exercise) intervention in older adults in the intervention group (37).

The small sample size and the lack of a control group were limitations of this study that should be mentioned. A small sample size limits generalizability to a broader population, and a lack of a control group limits a direct comparative set of data in individuals receiving the intervention and those who did not, which does limit the inferences that can be made. However, in spite of these limitations, this data provides information regarding the feasibility of delivering a lifestyle intervention in older adults with T2D and provides preliminary efficacy data for the effect of this intervention on frailty in this population. Secondly, the use of Fitbit to collect self-reported diet and physical activity may have led to imprecise reporting and may have allowed patient biases to affect the study's outcome; however, this limitation is inherent to all studies using selfreported measures. We attempted to minimize imprecision and bias by using validated objective assessments during the followup visit. Lastly, the COVID-19 pandemic did interfere with in-person group intervention as previously discussed.

\section{Conclusion and Implications}

Our study provides evidence for the feasibility of using a behavioral lifestyle intervention, enhanced with mobile health technology, for the goal of reducing frailty and improving T2D outcomes in community-dwelling overweight/obese older adults. Our results are particularly encouraging as they were observed despite our study being impacted by the COVID-19 pandemic. We demonstrated a high retention rate and observed that personal fitness technology (Fitbit activity tracker) is acceptable and maybe a valuable intervention tool for selfmonitoring in this population. Pre-frailty and frailty were common in our sample of older adults with T2D, in line with prior work showing that T2D and overweight/obesity are highly correlated with frailty. Detecting and managing frailty at its initial stages is ideal to improve healthspan and prevent disability and death. Further, screening for frailty and related measures, such as physical function and self-reported measures of health, may be beneficial for clinicians to identify high-risk patients who may benefit from the interventions to prevent or delay frailty. In the future, larger randomized controlled trials will be needed for definitive evidence of this intervention's efficacy to reduce frailty-in this high-risk population. Future, more robust studies may include a longer intervention phase and follow-up period, and more precise measurements of diet and physical activity, such as actimetry and 24-hour diet recall. In addition, cognitive assessments may also be important to examine the impact of such an intervention on cognitive function, in addition to frailty and physical function.

Acknowledgments: We would like to thank the University of Texas Health Science Center at San Antonio's School of Nursing for the use of the Center for Simulation Innovation, Office for Nursing Research Biobehavioral Lab, Center on Smart and Connected Health Technologies, and Geriatric Research, Education, and Clinical Center at the South Texas Veterans Health Care System, San Antonio, Texas for their support for this work.
Funding: This work was supported by the RL5 Mentored Research Career Development Award (Trainee: Rozmin Jiwani, Mentors: Sara Espinoza, Jing Wang) through the San Antonio Claude D. Pepper Older Americans Independence Center (P30AG044271) and the United States Department of Education through Title V Grant P031S150048 (Trainees: Edward Monk, Chandler Bess, Mentors: Darpan Patel, Rozmin Jiwani). This study was also supported by research infrastructure from the Geriatric Research, Education, and Clinical Center at the South Texas Veterans Health Care System.

Conflicts of Interest: There were no conflicts of interest declared by the authors for this study

Ethics declaration: The University of Texas Health Science Center's Institutional Review Board for the protection of human participants approved all procedures.

\section{References}

1. Fried LP, Tangen CM, Walston J, Newman AB, Hirsch C, Gottdiener J, et al. Frailty in older adults: evidence for a phenotype. J Gerontology A Biol Sci Med Sci. 2001;56(3):M146-56.

2. Collard RM, Boter H, Schoevers RA, Oude Voshaar RC. Prevalence of frailty in community-dwelling older persons: a systematic review. J Am Geriatr Soc. 2012;60(8):1487-92.

3. Assar ME, Laosa O, Rodríguez Mañas L. Diabetes and frailty. Curr Opin Clin Nutr Metab Care. 2019;22(1):52-7.

4. Espinoza SE, Jung I, Hazuda H. Frailty transitions in the San Antonio Longitudinal Study of Aging. J Am Geriatr Soc. 2012;60(4):652-60.

5. Li G, Prior JC, Leslie WD, Thabane L, Papaioannou A, Josse RG, et al. Frailty and Risk of Fractures in Patients With Type 2 Diabetes. Diabetes Care. 2019;42(4):507-13.

6. Morley JE, Malmstrom TK, Rodriguez-Manas L, Sinclair AJ. Frailty, sarcopenia and diabetes. JAMDA. 2014;15(12):853-9.

7. Ulley J, Abdelhafiz AH. Frailty predicts adverse outcomes in older people with diabetes. Practitioner. 2017;261(1800):17-20.

8. Espinoza SE, Jiwani R, Wang J, Wang CP. Review of Interventions for the Frailty Syndrome and the Role of Metformin as a Potential Pharmacologic Agent for Frailty Prevention. Clinical Therapeutics. 2019;41(3):376-86.

9. Pi-Sunyer X, Blackburn G, Brancati FL, Bray GA, Bright R, Clark JM, et al. Reduction in weight and cardiovascular disease risk factors in individuals with type 2 diabetes: one-year results of the look AHEAD trial. Diabetes Care. 2007;30(6):1374-83.

10. Wing RR. Long-term effects of a lifestyle intervention on weight and cardiovascular risk factors in individuals with type 2 diabetes mellitus: four-year results of the Look AHEAD trial. Arch Intern Med 2010;170(17):1566-75.

11. Look Ahead Research Group. Effect of a long-term behavioural weight loss intervention on nephropathy in overweight or obese adults with type 2 diabetes: a secondary analysis of the Look AHEAD randomised clinical trial. Lancet Diabetes Endocrinol. 2014;2(10):801-9.

12. Rejeski WJ, Bray GA, Chen SH, Clark JM, Evans M, Hill JO, et al. Aging and physical function in type 2 diabetes: 8 years of an intensive lifestyle intervention. J Gerontology A Biol Sci Med Sci. 2015;70(3):345-53.

13. Rejeski WJ, Ip EH, Bertoni AG, Bray GA, Evans G, Gregg EW, et al. Lifestyle change and mobility in obese adults with type 2 diabetes. N Engl J Med. 2012;366(13):1209-17.

14. Redmon JB, Bertoni AG, Connelly S, Feeney PA, Glasser SP, Glick H, et al. Effect of the look AHEAD study intervention on medication use and related cost to treat cardiovascular disease risk factors in individuals with type 2 diabetes. Diabetes Care. 2010;33(6):1153-8.

15. Espeland MA, Glick HA, Bertoni A, Brancati FL, Bray GA, Clark JM, et al. Impact of an intensive lifestyle intervention on use and cost of medical services among overweight and obese adults with type 2 diabetes: the action for health in diabetes. Diabetes Care. 2014;37(9):2548-56.

16. Simpson FR, Pajewski NM, Nicklas B, Kritchevsky S, Bertoni A, Ingram F, et al. Impact of Multidomain Lifestyle Intervention on Frailty Through the Lens of Deficit Accumulation in Adults with Type 2 Diabetes Mellitus. J Gerontology A Biol Sci Med Sci. 2019.

17. Agarwal S, LeFevre AE, Lee J, L'Engle K, Mehl G, Sinha C, et al. Guidelines for reporting of health interventions using mobile phones: mobile health (mHealth) evidence reporting and assessment (mERA) checklist. BMJ (Clinical research ed). 2016;352:i1174.

18. Anderson M, Perrin A. Technology Adoption Climbs Among Older Adults. Washington, DC: Pew Research Center. 2017. https://www. pewresearch.org/internet/2017/05/17/technology-use-among-seniors/ 
19. Elavsky S, Knapova L, Klocek A, Smahel D. Mobile Health Interventions for Physical Activity, Sedentary Behavior, and Sleep in Adults Aged 50 Years and Older: A Systematic Literature Review. J Aging Phys Act. 2019;27(4):565-93.

20. Espinoza SE, Orsak B, Wang CP, MacCarthy D, Kellogg D, Powers $\mathrm{B}$, et al. An Individualized Low-Intensity Walking Clinic Leads to Improvement in Frailty Characteristics in Older Veterans. J Frailty Aging. 2019;8(4):205-9.

21. Jiwani R, Wang J, Berndt A, Ramaswamy P, Mathew Joseph N, Du Y, et al. Changes in Patient-Reported Outcome Measures With a TechnologySupported Behavioral Lifestyle Intervention Among Patients With Type 2 Diabetes: Pilot Randomized Controlled Clinical Trial. JMIR Diabetes. 2020;5(3):e19268.

22. Wang J, Cai C, Padhye N, Orlander P, Zare M. A Behavioral Lifestyle Intervention Enhanced With Multiple-Behavior Self-Monitoring Using Mobile and Connected Tools for Underserved Individuals With Type 2 Diabetes and Comorbid Overweight or Obesity: Pilot Comparative Effectiveness Trial. JMIR mHealth and uHealth. 2018;6(4):e92.

23. Jiwani R, Wang C-P, Orsak B, MacCarthy D, Kellogg D, Powers B, et al. A geriatrics walking clinic improves hemoglobin A1c and time gait in veterans with type 2 diabetes. Geriatric Nursing. 2020.

24. Moore CG, Carter RE, Nietert PJ, Stewart PW. Recommendations for planning pilot studies in clinical and translational research. Clin Transl Sci. 2011;4(5):332-7.

25. Folsom AR, Jacobs DR, Jr., Caspersen CJ, Gomez-Marin O, Knudsen J. Test-retest reliability of the Minnesota Leisure Time Physical Activity Questionnaire. J Chronic Dis. 1986;39(7):505-11.

26. Espinoza SE, Hazuda HP. Frailty in older Mexican-American and European-American adults: is there an ethnic disparity? J Am Geriatr Soc. 2008;56(9):1744-9.

27. Guralnik JM, Ferrucci L, Simonsick EM, Salive ME, Wallace RB. Lowerextremity function in persons over the age of 70 years as a predictor of subsequent disability. N Engl J Med. 1995;332(9):556-61.

28. Guralnik JM, Simonsick EM, Ferrucci L, Glynn RJ, Berkman LF, Blazer DG, et al. A short physical performance battery assessing lower extremity function: association with self-reported disability and prediction of mortality and nursing home admission. J Gerontology A Biol Sci Med Sci. 1994;49(2):M85-94.
29. Cella D, Riley W, Stone A, Rothrock N, Reeve B, Yount S, et al. The Patient-Reported Outcomes Measurement Information System (PROMIS) developed and tested its first wave of adult self-reported health outcome item banks: 2005-2008. J Clin Epidemiol. 2010;63(11):1179-94.

30. Meek G, Ozgur C, Dunning K. Comparison of the t vs. Wilcoxon SignedRank Test for Likert Scale Data and Small Samples. J. Mod. Appl. Stat. Methods. 2007;6(1).

31. Rye Hanton C, Kwon Y-J, Aung T, Whittington J, High RR, Goulding EH, et al. Mobile Phone-Based Measures of Activity, Step Count, and Gait Speed: Results From a Study of Older Ambulatory Adults in a Naturalistic Setting. JMIR mHealth and uHealth. 2017;5(10):e104.

32. Burke LE, Wang J, Sevick MA. Self-monitoring in weight loss: a systematic review of the literature. J Am Diet Assoc. 2011;111(1):92-102.

33. Jiwani R, Dennis B, Bess C, Monk S, Meyer K, Wang J, et al. Assessing acceptability and patient experience of a behavioral lifestyle intervention using fitbit technology in older adults to manage type 2 diabetes amid COVID-19 pandemic: A focus group study. Geriatric Nursing. 2021;42(1):57-64.

34. Pritchard JM, Kennedy CC, Karampatos S, Ioannidis G, Misiaszek B Marr S, et al. Measuring frailty in clinical practice: a comparison of physical frailty assessment methods in a geriatric out-patient clinic. BMC Geriatr. 2017;17(1):264

35. Lauretani F, Ticinesi A, Gionti L, Prati B, Nouvenne A, Tana C, et al Short-Physical Performance Battery (SPPB) score is associated with falls in older outpatients. Aging Clin Exp Res. 2019;31(10):1435-42.

36. Bandinelli S, Lauretani F, Boscherini V, Gandi F, Pozzi M, Corsi AM, et al. A randomized, controlled trial of disability prevention in frail older patients screened in primary care: the FRASI study. Design and baseline evaluation. Aging Clin Exp Res. 2006;18(5):359-66.

37. Rodriguez-Mañas L, Laosa O, Vellas B, Paolisso G, Topinkova E, OlivaMoreno J, et al. Effectiveness of a multimodal intervention in functionally impaired older people with type 2 diabetes mellitus. J Cachexia Sarcopenia Muscle. 2019;10(4):721-33.

How to cite this article: R. Jiwani, J. Wang, C. Li et al. A Behavioral Lifestyle Intervention to Improve Frailty in Overweight or Obese Older Adults with Type 2 Diabetes: A Feasibility Study. J Frailty Aging 2022;11(1)74-82; http://dx.doi org/10.14283/jfa.2021.17 